\title{
A Phase 1 study of UCN-01 in combination with irinotecan in patients with resistant solid tumor malignancies
}

\author{
Paula M. Fracasso $\cdot$ Kerry J. Williams $\cdot$ Ronald C. Chen $\cdot$ Joel Picus $\cdot$ Cynthia X. Ma $\cdot$ Matthew J. Ellis • \\ Benjamin R. Tan · Timothy J. Pluard · Douglas R. Adkins $\cdot$ Michael J. Naughton · Janet S. Rader • \\ Matthew A. Arquette $\cdot$ James W. Fleshman $\cdot$ Allison N. Creekmore $\cdot$ Sherry A. Goodner $\cdot$ \\ Lisa P. Wright $\cdot$ Zhanfang Guo $\cdot$ Christine E. Ryan $\cdot$ Yu Tao $\cdot$ Eliane M. Soares $\cdot$ Shi-rong Cai $\cdot$ \\ Li Lin · Janet Dancey $\cdot$ Michelle A. Rudek • Howard L. McLeod · Helen Piwnica-Worms
}

Received: 14 April 2010/Accepted: 11 July 2010/Published online: 8 August 2010

(C) The Author(s) 2010. This article is published with open access at Springerlink.com

\begin{abstract}
Purpose UCN-01 (7-hydroxystaurosporine) is a multitargeted protein kinase inhibitor that exhibits synergistic activity with DNA-damaging agents in preclinical studies. We conducted a Phase I study to determine the maximumtolerated dose (MTD), dose-limiting toxicity (DLT), pharmacokinetic, and pharmacodynamic effects of UCN01 and irinotecan in patients with resistant solid tumors.

Experimental design Patients received irinotecan $\left(75-125 \mathrm{mg} / \mathrm{m}^{2}\right.$ IV on days $\left.1,8,15,22\right)$ and UCN-01 $\left(50-90 \mathrm{mg} / \mathrm{m}^{2} \mathrm{IV}\right.$ on day 2 and $25-45 \mathrm{mg} / \mathrm{m}^{2}$ on day 23
\end{abstract}

Preliminary results of this study were published in abstract form in $\mathrm{J}$ Clin Oncol 2004;22:702s and Clin Cancer Res 2005;11:8,985s and presented at the AACR-NCI-EORTC Molecular Targets and Therapeutics 2005.

M. A. Arquette: Is deceased.

H. L. McLeod and H. Piwnica-Worms contributed equally to this work.

Electronic supplementary material The online version of this article (doi:10.1007/s00280-010-1410-1) contains supplementary material, which is available to authorized users.

P. M. Fracasso - K. J. Williams - R. C. Chen - J. Picus ·

C. X. Ma · M. J. Ellis · B. R. Tan · T. J. Pluard .

D. R. Adkins - M. J. Naughton - M. A. Arquette

A. N. Creekmore - S. A. Goodner - L. P. Wright - Z. Guo ·

Y. Tao · E. M. Soares · S. Cai · L. Lin ·

H. L. McLeod · H. Piwnica-Worms

Department of Internal Medicine, Alvin J. Siteman Cancer Center and Washington University School of Medicine,

St. Louis, MO, USA

J. S. Rader

Department of Obstetrics and Gynecology,

Alvin J. Siteman Cancer Center and Washington University

School of Medicine, St. Louis, MO, USA and subsequent doses) every 42 days. Blood for pharmacokinetics of UCN-01 and irinotecan, and blood, normal rectal mucosa, and tumor biopsies for pharmacodynamic studies were obtained.

Results Twenty-five patients enrolled to 5 dose levels. The MTD was irinotecan $125 \mathrm{mg} / \mathrm{m}^{2}$ on days $1,8,15,22$ and UCN-01 $70 \mathrm{mg} / \mathrm{m}^{2}$ on day 2 and $35 \mathrm{mg} / \mathrm{m}^{2}$ on day 23 . DLTs included grade 3 diarrhea/dehydration and dyspnea. UCN-01 had a prolonged half-life and a low clearance rate. There was a significant reduction in $\mathrm{SN}-38 \mathrm{C}_{\max }$ and aminopentanocarboxylic acid (APC) and SN-38 glucuronide half-lives. Phosphorylated ribosomal protein S6 was reduced in blood, normal rectal mucosa, and tumor biopsies at $24 \mathrm{~h}$ post-UCN-01. Two partial responses were observed in women with ER, PgR, and HER2-negative breast cancers (TBNC). Both tumors were defective for p53. Twelve patients had stable disease (mean duration 18 weeks, range 7-30 weeks).

Conclusion UCN-01 and irinotecan demonstrated acceptable toxicity and target inhibition. Anti-tumor activity was observed and a study of this combination in women with TNBC is underway.

\section{J. W. Fleshman}

Department of Surgery, Alvin J. Siteman Cancer Center and Washington University School of Medicine,

St. Louis, MO, USA

C. E. Ryan · S. Cai · H. Piwnica-Worms

Department of Cell Biology and Physiology,

Alvin J. Siteman Cancer Center and Washington University

School of Medicine, St. Louis, MO, USA

C. E. Ryan - S. Cai · H. Piwnica-Worms

Howard Hughes Medical Institute, Bethesda, MD, USA 
Keywords Phase 1 - Irinotecan - UCN-01 - Chk1 . Ribosomal protein S6

\section{Introduction}

Originally isolated as a protein kinase $\mathrm{C}$ inhibitor, $\mathrm{UCN}-01$ has been shown to inhibit several protein kinases, including Chk1, PDK1, and the cell cycle regulatory kinases, Cdk2, Cdk4 and Cdk6 [7, 18, 46, 59-62]. As a single agent, $\mathrm{UCN}-01$ is capable of inducing cell cycle arrest at the G1/S-border [3-6, 26, 39, 51, 66, 67] and has anti-tumor effects in several NCI human tumor cell lines and xenograft models [1, 3, 9, 27, 40, 49, 50]. When combined with DNA-damaging agents, UCN-01 is capable of abrogating S- and G2-checkpoints [6, 7, 13, 35, 67]. This is thought to be due to UCN-01's ability to inhibit the Chk1 protein kinase, critical to the regulation of the S- and G2-checkpoints in response to DNA damage in cells lacking a G1 checkpoint due to TP53 mutations [25]. With S- and G2-checkpoint abrogation by Chk1 inhibition, p53-deficient cancer cells fail to arrest and undergo mitotic catastrophe and eventually apoptosis $[6,7,18,28,36,67,68]$. Inhibition of PDK1 may also be an important contributor to the anti-tumor activity of UCN-01 [46]. Breast cancer cell lines exposed to PDK1 inhibitors undergo cell death and exhibit reduced proliferation rates presumably through opposition of the PI3 K/AKT pathway [11,31]. As a potent inhibitor of both Chk1 and PDK1, UCN-01 has the potential to

\author{
J. Dancey \\ Cancer Therapy Evaluation Program, \\ National Cancer Institute, Bethesda, MD, USA
}

M. A. Rudek

Sidney Kimmel Comprehensive Cancer Center at Johns

Hopkins, Baltimore, MD, USA

P. M. Fracasso $(\square)$

Department of Medicine, University of Virginia,

P.O. Box 800716, Charlottesville, VA 22908, USA

e-mail: fracasso@virginia.edu

Present Address:

H. L. McLeod

Institute for Pharmacogenomics and Individualized Therapy,

University of North Carolina, Chapel Hill, NC, USA

Present Address:

R. C. Chen

Department of Radiation Oncology, University of North

Carolina, Chapel Hill, NC, USA

Present Address:

J. Dancey

High Impact Clinical Trials, The Ontario Institute

for Cancer Research, Toronto, ON, Canada target two important cellular processes that are frequently deregulated in cancer cells.

In Phase I clinical trials, as a single agent, UCN-01 has been evaluated using a 72 and $3 \mathrm{~h}$ infusion schedule $[12,47,48]$. Due to pharmacokinetic data demonstrating that UCN-01 binds tightly to $\alpha$-1-acid glycoprotein (AAG) resulting in a long half-life of several weeks, UCN-01 dosing was reduced by half after the first cycle in both schedules. This long plasma half-life in humans raised the question as to whether UCN-01 was actually bioavailable to tissue at the concentrations required for S/G2-checkpoint abrogation. In addition, UCN-01 was observed to have unusual DLTs including hyperglycemia with lactic acidosis, pulmonary toxicity (hypoxemia), nausea, vomiting and hypotension. Phase I studies of UCN-01 in combination with carboplatin, cisplatin, cytarabine, 5-fluorouracil, irinotecan, and topotecan have also been performed, and similar pharmacokinetics of UCN-01 and DLTs were observed [14, 19, 22, 30, 34, 41, 45].

We hypothesized that $\mathrm{UCN}-01$ and irinotecan would be an effective regimen in a broad range of refractory malignancies. Irinotecan (CPT-11, Camptosar $\left.{ }^{\circledR}\right)$, a semisynthetic analog of camptothecin, serves as a topoisomerase poison covalently binding with topoisomerase I in a cleavable complex with a single strand break in the DNA. In the presence of ongoing DNA replication, the drug-stabilized cleavable complex is converted into a double-strand break leading to severe DNA damage and eventual apoptosis. This anticancer agent has activity in several solid tumor malignancies [54]. Furthermore, synergism between camptothecin or SN-38, the active metabolite of irinotecan, and UCN-01 has been shown in multiple preclinical studies $[23,24,36,52$, 65]. We envisioned that UCN- 01 would both inhibit Chk 1 to abrogate checkpoint responses induced by irinotecan and inhibit PDK1 to induce apoptosis. In this Phase I study, we set out to determine the MTD, assess the safety and toxicity, and conduct pharmacokinetic and pharmacodynamic studies to further understand the molecular basis of $\mathrm{UCN}-01$ activity in combination with irinotecan.

\section{Patients and methods}

Patient eligibility

Patients at least 18 years of age were eligible for enrollment into the study if they had a histologically confirmed malignant solid tumor for which standard curative treatment did not exist or was no longer effective, measurable or evaluable disease, and an Eastern Cooperative Oncology Group (ECOG) performance status of 0-2. Laboratory criteria included absolute neutrophil count $\geq 1,500 / \mu 1$, platelet counts $\geq 100,000 / \mu \mathrm{l}$, serum creatinine $\leq 1.5 \times$ upper limit of normal (ULN), aspartate aminotransferase and alanine 
aminotransferase $\leq 3 \times$ ULN, and total bilirubin $\leq 1.5 \times$ ULN. Prior chemotherapy or radiotherapy must have been completed at least 4 weeks prior to treatment. Patients with brain metastasis, known sensitivity to UCN-01 or irinotecan, insulin-dependant diabetes mellitus or uncontrolled intercurrent illness, diagnosis of Gilbert's disease, or chronic unconjugated hyperbilirubinemia were excluded. Initially, all patients on this trial were required to have a DLCO $\geq 60 \%$ and oxygen saturation $\geq 90 \%$ on room air at rest and after a 6-min walk. However, to increase eligibility, the requirement to obtain a DLCO was removed. The Cancer Therapy Evaluation Program (P5582), Division of Cancer Treatment and Diagnosis, National Cancer Institute (CTEP, $\mathrm{NCI}$ ) and the Washington University Human Research Protection Office approved this protocol. All patients provided written informed consent prior to study entry.

\section{Treatment plan and study design}

Patients received irinotecan $\left(\right.$ Camptosar $^{\circledR}$, Pfizer Inc., New York, NY) as a 90-min intravenous infusion on days 1, 8, 15, and 22 and UCN-01 (Kyowa Hakko Kogyo Co., Shizuoka, Japan and supplied by CTEP, NCI) as a 3-h continuous intravenous infusion on days 2 and 23 every 42 days as proposed in the dose escalation schema in Table 1. The first dose of UCN-01 (day 1 of cycle 1) was twice that used in remaining doses due to the prolonged half-life of UCN-01.

Three to six patients were enrolled at each dose level. Toxicities were graded in accordance with the National Cancer Institute Common Terminology Criteria for Adverse Events (NCI CTC) version 2.0. Dose-limiting toxicity (DLT) was determined by toxicity observed during cycle 1. DLT was defined as grade 4 neutropenia of any duration, febrile neutropenia, grade 3 or 4 thrombocytopenia, grade 3 and 4 non-hematologic toxicity including grade 3 diarrhea lasting more than $24 \mathrm{~h}$ despite optimal supportive medications, grade 4 vomiting despite optimal antiemetic therapy, and any toxicity causing a delay of $>14$ days. Grade 3 nausea, vomiting, and hyperglycemia

Table 1 Dose escalation schema

\begin{tabular}{lllll}
\hline $\begin{array}{l}\text { Dose } \\
\text { level }\end{array}$ & $\begin{array}{l}\text { No. of } \\
\text { patients }\end{array}$ & $\begin{array}{l}\text { Irinotecan } \\
\left(\mathrm{mg} / \mathrm{m}^{2}\right)\end{array}$ & $\begin{array}{l}\mathrm{UCN}-01 \\
\left(\mathrm{mg} / \mathrm{m}^{2}\right)^{*}\end{array}$ & $\begin{array}{l}\text { No. of } \\
\text { DLTs }\end{array}$ \\
\hline 1 & 3 & 75 & 50 & 0 \\
2 & 3 & 75 & 70 & 0 \\
3 & 6 & 100 & 70 & 1 \\
4 & $8^{\#}$ & 125 & 70 & 1 \\
5 & 5 & 125 & 90 & 3 \\
\hline
\end{tabular}

* Subsequent doses of UCN-01 were half the dose of this first dose

\# Two patients did not complete the first cycle were not considered a DLT. If a DLT occurred in one of three patients, up to 3 additional patients were treated at that dose level. If two patients at this dose level experienced a DLT, enrollment would be terminated. If only one of six patients experienced a DLT, dose escalation proceeded. The maximum-tolerated dose (MTD) was defined as one dose level lower than that with which at least two patients experienced a DLT.

Dose modifications for irinotecan and UCN-01 included dose delays and reductions. Treatment with irinotecan alone or in combination with UCN-01 was delayed until the following criteria were met: the absolute neutrophil count (ANC) $\geq 1,500$ cells $/ \mu \mathrm{L}$, platelets $\geq 100,000$ cells $/ \mu \mathrm{L}$, hemoglobin $\geq 10$, and all other treatment-related toxicities $\leq$ grade 1 . When treatment resumed, irinotecan was reduced by $25 \mathrm{mg} / \mathrm{m}^{2}$ for grade 3 and $50 \mathrm{mg} / \mathrm{m}^{2}$ for grade 4 hematologic and non-hematologic toxicities. For grade 2 hematologic and non-hematologic toxicities, irinotecan was reduced by $25 \mathrm{mg} / \mathrm{m}^{2}$ in the current cycle of treatment and returned to normal dosage in subsequent cycles, provided the above criteria were met. For grade 2 hyperglycemia and grade 3 and 4 hyperglycemia and nausea and vomiting, UCN-01 was also reduced by $10 \mathrm{mg} / \mathrm{m}^{2}$ for all subsequent treatments. For irreversible grade 2 cardiopulmonary toxicity or grade 3 or 4 cardiopulmonary or hepatic toxicity, patients were removed from study. If irinotecan was delayed for toxicity on day 22 and subsequently administered on day 29, the UCN-01 was also delayed and given on day 30 . Any treatment-related toxicity requiring a delay of $>14$ days in the first cycle was considered dose limiting. If, after the appropriate dose reductions, a treatment-related toxicity required a delay of $>14$ days in subsequent cycles, the patient was taken off study.

Antitumor response was evaluated by physical examination and/or imaging pre-study and every cycle. Responses were defined by Response Evaluation Criteria in Solid Tumors (RECIST) [64].

\section{Pharmacokinetic monitoring}

Plasma samples were obtained to determine the pharmacokinetics of total UCN-01 and irinotecan and its metabolites, SN-38, SN-38 glucuronide (SN-38G), and APC. Samples were obtained prior to UCN-01 infusion, $5 \mathrm{~min}$ before the end of the infusion, $20-26 \mathrm{~h}$ post-UCN-01 infusion, and days 8, 15, and 22 for patients in Dose Levels 1-3. Sampling was extended for patients in Dose Levels 4 and 5 to include the additional samples: $0.5,1.5 \mathrm{~h}$, and post-infusion at $0.5,2,4,24,48 \mathrm{~h}$. Plasma samples were analyzed using modifications to previous analytical assays consisting of high-performance liquid chromatography (HPLC) with fluorescence detection [12, 22]. UCN-01 plasma quantitative range was $0.2-60 \mu \mathrm{g} / \mathrm{mL}$. 
For irinotecan, plasma samples were obtained in cycle 1 prior to irinotecan infusion, and at $0.25,1.5,2.25,3.0,5.5$, $8.5,24 \mathrm{~h}$ (prior to the start of UCN-01), $27 \mathrm{~h}$ (prior to the end of the UCN-01 infusion), and $48 \mathrm{~h}$ after the start of the irinotecan infusion on the first and second weekly doses of irinotecan. Plasma samples were analyzed for irinotecan and its metabolites using a modified HPLC technique with fluorescence detection as previously described [43].

Pre-treatment AAG was determined using a nephelometric assay (Focus Diagnostics, Inc., Cypress, CA). The normal range of AAG using this assay is $36-126 \mathrm{mg} / \mathrm{dL}$.

Individual plasma concentrations of UCN-01, irinotecan and metabolites were analyzed using non-compartmental methods as implemented in the computer software program WinNonlin version 5.0 (Pharsight, Inc., Mountain View, CA) $[17,22]$. ANOVA was used to determine the association among patient demographics and UCN-01 and irinotecan exposure and worst grade of toxicity during course 1 . The method of Tukey-Kramer was used to adjust for multiple comparisons of mean values. Statistical analysis was done using JMP Statistical Discovery Software version 3.2.6 (SAS Institute, Cary, NC). The a priori level of significance was $P<0.05$.

\section{Pharmacodynamic studies}

Peripheral blood mononuclear cells (PBMC) were collected at baseline (day 1), $24 \mathrm{~h}$ post-irinotecan but prior to UCN01 (day 2), 24 h post-UCN-01 treatment (day 3) and on day 8 prior to the second irinotecan treatment during cycle 1 for 24 patients for Western blot analysis of phosphorylated ribosomal protein S6. Samples from patient \#7 were excluded from analysis due to poor quality. The PBMC were lysed in loading buffer (10\% glycerol, $2 \%$ sodium dodecyl sulfate, $0.0625 \mathrm{M}$ Tris- $\mathrm{HCl}, \mathrm{pH} 6.8$ and $5 \%$ $\beta$-mercaptoethanol), boiled for $10 \mathrm{~min}$, sonicated for $5 \mathrm{~min}$ in a water bath sonicator, re-boiled for $3 \mathrm{~min}$, and pelleted at $16,000 \mathrm{~g}$ for $5 \mathrm{~min}$. Supernatants were assayed for protein concentration, and $60 \mu \mathrm{g}$ of total cellular protein was run on Criterion gels (Bio-Rad Laboratories, Hercules, CA) after adding bromophenol blue. Proteins were transferred to PVDF and probed with phospho-S6 ribosomal protein Ser240/244, S6 ribosomal protein (Cell Signaling Technology, Inc., Danver, MA), and actin (Sigma Chemical Co., St. Louis, MO). Blots were developed using ECL detection reagent (GE Healthcare, Piscataway, $\mathrm{NJ}$ ), and proteins were quantitated using ImageJ [2]. The ratio of phosphorylated S6 to total S6 protein was determined for each sample.

Biopsies of normal rectal mucosa performed by colonoscopy were obtained at baseline and $24 \mathrm{~h}$ post-UCN-01 for immunohistochemistry (IHC) of phosphorylated S6 on patients from Dose Levels 1-3. However, due to slow accrual to this study, thought secondary to these biopsies, this study requirement was made optional for patients on Dose Levels 4 and 5. Skin punch biopsies ( $4 \mathrm{~mm}$ ) from two women (patients 14 and 24) from Dose Level 4 with metastatic breast cancer to the skin of the chest wall were also obtained at baseline and $24 \mathrm{~h}$ post-UCN-01 for IHC of phosphorylated S6 (Cell Signaling Technology, Danver, MA), Cdc2p34 Tyr15 (Santa Cruz Biotechnology, Inc, Santa Cruz, CA), Chk1 Ser317 and cleaved caspase 3 (Cell Signaling Technology, Inc., Danvers, MA), phosphohistone H3, and histone H2AX Ser139 (Millipore Corporation, Billerica, MA). Both rectal mucosa and tumor biopsies were fixed in formalin, processed for hematoxylin and eosin staining, and reviewed by a pathologist to confirm cellularity prior to IHC studies. Immunodetection was performed using the Histostain-Plus Kits (Zymed Laboratories Inc, South San Francisco, CA). Blocking solution and rabbit IgG were used as negative controls for the IHC. The intensity of staining was graded from 0 to 3 , zero being no staining, (1) light, (2) intermediate, and (3) strong staining. The distribution of staining (percentage of tumor cells staining positive) was also assessed.

Tumor genomic DNA was isolated from three patients (patients 14, 22, and 24) with metastatic breast cancer (two from the baseline chest wall tumor biopsies and one from the archival primary breast tumor surgical specimen) and analyzed for TP53 mutation by direct nucleotide sequencing of polymerase chain products of exons 4-9. Due to the quality of tissue samples, TP53 sequencing was successful in only two (from patients 14 and 24) of the three specimens. IHC of p53 (Santa Cruz Biotechnology, Inc, Santa Cruz, CA) (1:200 dilution, overnight) was performed on the third patient sample (patient 22), using the same detection methodology described previously.

Tumor genomic DNA from the chest wall breast tumor biopsy of two patients was analyzed for TP53 mutations by direct nucleotide sequencing of polymerase chain products of each of the exons 4-9.

For Western blot analyses of pS6/S6 in PBMC, a natural $\log$ transformation of the pS6/S6 ratio was required for a normal distribution necessary for the application of standard parametric tests. After log transformation, a paired $t$ test and one-way ANOVA were used to assess the differences among different time points (days 1, 2, 3, 8). Homogeneity of pS6/S6 ratio variance was assessed by Levene's test, and the variances were found to be equal.

\section{Results}

Patient characteristics

Between January 2002 and January 2007, 25 patients with advanced resistant solid tumor malignancies were enrolled. 
Patient characteristics are summarized in Table 2. The majority of patients had breast or colorectal cancer and had multiple prior therapies.

\section{Treatment administration}

Twenty-three of the 25 patients completed at least one cycle of treatment (range 1-5) for a total of 52 cycles. Two patients at Dose Level 4 did not complete the first cycle of therapy due to progressive disease. As per study design, three patients were treated at each dose level (Table 1). Patients at Dose Levels 1 and 2 were treated without a DLT. At Dose Level 3, one of the first three patients had grade 3 dyspnea, which was a DLT. Three additional patients were added with no further DLTs. At Dose Level 4 , four patients (one patient did not complete a full cycle due to progressive disease) were treated without DLT. At Dose Level 5, one of the first 3 patients had a DLT consisting of grade 3 diarrhea with accompanied dehydration and grade 3 hypokalemia and neutropenia. Thus, two additional patients were added. One of these patients developed a DLT consisting of grade 3 diarrhea. The other patient developed grade 3 hypophosphatemia and grade 3 nausea and vomiting. As a result, no further patients were treated at Dose Level 5 and 4 additional patients were added to Dose Level 4 . Of the first 3 additional patients, one patient received one weekly treatment with irinotecan and UCN-01 and developed grade 4 vomiting and grade 3 dehydration (a DLT). This patient was taken off study due

Table 2 Patient characteristics

\begin{tabular}{ll}
\hline Characteristics & $\begin{array}{l}\text { No. patients } \\
(n=25)\end{array}$ \\
\hline Age (y) & $54(19-75)$ \\
Mean (range) & \\
Sex & $15: 10$ \\
Female: male & \\
ECOG performance status & 13 \\
0 & 8 \\
1 & 4 \\
2 & \\
Tumor types & 10 \\
Breast & 6 \\
Colorectal & 2 \\
Unknown primary & 7 \\
Other (anus, esophagus, head \& neck, & \\
$\quad$ osteosarcoma, ovary, pancreas, prostate) & \\
Prior therapy & 25 \\
Chemotherapy & $3.5(1-6)$ \\
Mean no. regimens (range) & 16 \\
Radiotherapy &
\end{tabular}

to progressive disease, and a fourth patient was treated without complication. Therefore, the MTD was considered Dose Level 4 (irinotecan $125 \mathrm{mg} / \mathrm{m}^{2}$ on days $1,8,15$, and 22 and UCN-01 $70 \mathrm{mg} / \mathrm{m}^{2}$ on day 2 and $35 \mathrm{mg} / \mathrm{m}^{2}$ on day 23 and all subsequent doses on a 42-day cycle).

\section{Toxicity}

The most common hematologic toxicity was neutropenia with $48 \%$ of patients exhibiting grade 2 or 3 neutropenia during the first cycle (Table 3 ). One patient at Dose Level 3 had a 13-day delay due to grade 3 neutropenia. One patient at Dose Level 1, two patients each at Dose Levels 3 and 4, and 3 patients at Dose Level 5 had a 1-week delay due to grade 2 neutropenia. The most common nonhematologic toxicities were nausea/vomiting $(60 \%)$, fatigue/malaise (48\%), diarrhea (48\%), and hyperglycemia (44\%). Other toxicities included anorexia, dehydration (associated with nausea/vomiting or diarrhea), dyspnea, hypotension, abdominal pain or cramping, stomatitis (Table 3). UCN-01 $\mathrm{C}_{\max }$ was related to worst grade of hypotension during course $1(P=0.04)$, but there was no clear correlation of increased exposure to increased grade of toxicity. Irinotecan, and not the metabolite day 1 AUC, was related to the worst grade of diarrhea $(P=0.02)$ with increasing irinotecan exposure resulting in increased toxicity. Other measures of drug exposure for either irinotecan or UCN-01 were not correlated with other toxicities.

\section{Pharmacokinetics}

Blood sampling for pharmacokinetics was obtained on 25 patients for UCN-01 and on 23 patients for irinotecan.

A summary of the UCN-01 pharmacokinetic variables is presented in Table 4. At the MTD (Dose Level 4), the recommended dose level for this current study, the mean values for peak plasma concentration $\left(C_{\max }\right)$, and area under the plasma concentration-time curve (AUC $\left.\mathrm{AO}_{[0-\infty]}\right)$ were $15.4 \mu \mathrm{g} / \mathrm{mL}$ and $4,793 \mu \mathrm{g} * \mathrm{~h} / \mathrm{mL}$, and values for these exposure variables varied 2.1- and 2.7-fold, respectively. These parameters are comparable to those reported from the Phase 1 study of 3-h infusion of UCN-01 as a single agent $[12,47]$. Despite a statistically significant decrease in $\mathrm{C}_{\max }(P=0.01)$ with increasing dose level, there was no difference in the $\mathrm{AUC}_{[0-\infty]}(P=0.29)$ or systemic clearance $(P=0.10)$ as a function of dose level.

The relationships between pre-treatment AAG and UCN-01 pharmacokinetic variables were assessed to elucidate clinical variables that may be related to UCN-01 exposure. Pre-treatment AAG was determined in all patients with median level of $119 \mathrm{mg} / \mathrm{dL}$ (range 35-287 mg/dL). There is a significant relationship between total UCN-01 clearance and AAG concentration and with increasing AAG 
Table 3 Incidence of toxicities in cycle 1

\begin{tabular}{|c|c|c|c|c|c|c|c|c|c|c|c|c|c|c|c|c|c|c|c|c|}
\hline \multirow{3}{*}{$\begin{array}{l}\text { Adverse event } \\
\text { Grade of adverse } \\
\text { event }\end{array}$} & \multicolumn{20}{|c|}{ Dose level } \\
\hline & \multicolumn{4}{|c|}{$1(n=3)$} & \multicolumn{4}{|c|}{$2(n=3)$} & \multicolumn{4}{|c|}{$3(n=6)$} & \multicolumn{4}{|c|}{$4(n=8)$} & \multicolumn{4}{|c|}{$5(n=5)$} \\
\hline & 1 & 2 & 3 & 4 & 1 & 2 & 3 & 4 & 1 & 2 & 3 & 4 & 1 & 2 & 3 & 4 & 1 & 2 & 3 & 4 \\
\hline \multicolumn{21}{|l|}{ Blood/bone marrow } \\
\hline Hemoglobin & 1 & 1 & & & 1 & 1 & & & & 4 & $1 *$ & & 3 & 3 & 1 & & 3 & 2 & & \\
\hline Neutrophils/granulocytes & & 1 & & & 1 & 1 & & & 1 & 2 & $1 *$ & & & 2 & 1 & & & 3 & $1^{\#}$ & \\
\hline Platelets & & & & & & & & & & & & & 1 & & & & & & & \\
\hline \multicolumn{21}{|l|}{ Constitutional symptoms } \\
\hline Fatigue/malaise & 1 & 1 & & & 1 & 1 & & & 2 & 1 & $1 *$ & & 2 & & & & & 2 & & \\
\hline \multicolumn{21}{|l|}{ Cardiovascular } \\
\hline Hypotension & & & & & & & & & & & & & & 1 & & & & 2 & & \\
\hline \multicolumn{21}{|l|}{ Gastrointestinal } \\
\hline Anorexia & & 1 & & & 1 & 1 & & & 1 & 1 & & & 1 & & & & & & & \\
\hline Dehydration & & & & & & & & & & & & & & & $1 * *$ & & & & $1^{\#}$ & \\
\hline Diarrhea & 2 & & & & 1 & 1 & & & 2 & 2 & & & & 1 & & & 1 & & $2^{\delta \#}$ & \\
\hline Nausea/vomiting & & 1 & & & 2 & 1 & & & 1 & 1 & & & 2 & & 1 & $1 * *$ & 2 & 1 & $2^{++}$ & \\
\hline Stomatitis & 1 & & & & & & & & 2 & & & & & & & & & & & \\
\hline \multicolumn{21}{|l|}{ Metabolic/laboratory } \\
\hline Hyperglycemia & & & & & & 2 & & & 1 & 1 & & & 1 & 2 & 1 & & & 3 & & \\
\hline Hypocalcemia & & & & & & & & & & & & & 2 & & & & 1 & 2 & & \\
\hline Hypokalemia & & & & & & & & & 1 & & & & 2 & & & & & & $1^{\#}$ & \\
\hline Hypophosphatemia & & & & & & & & & 1 & 1 & & & & & & & & & $1^{++}$ & \\
\hline Pain & 1 & & & & 3 & & & & 1 & 1 & & & 1 & 1 & & & & 1 & & \\
\hline \multicolumn{21}{|l|}{ Pulmonary } \\
\hline Dyspnea & & & & & & 1 & & & & & $1 *$ & & & 1 & & & & & & \\
\hline
\end{tabular}

* Dose Level 3: One patient had grade 3 dyspnea (DLT) with grade 3 fatigue, anemia and neutropenia

** Dose Level 4: One patient had grade 3 dehydration (DLT) and grade 4 vomiting

Dose Level 5: ${ }^{\delta}$ One patient had grade 3 diarrhea (DLT), \# one patient had grade 3 diarrhea (DLT) with grade 3 dehydration, hypokalemia, and neutropenia, and ${ }^{++}$one patient had grade 3 hypophosphatemia (DLT) and grade 3 nausea/vomiting

concentrations, clearance values decreased linearly $\left(R^{2}=\right.$ $0.362 ; P=0.004)$.

A summary of the irinotecan pharmacokinetic variables is presented in Table 4 . When using a paired t-test to compare AUC, $\mathrm{C}_{\max }$, and $\mathrm{T}_{1 / 2}$ on both day 1 and day 8 , there was a significant increase in $\mathrm{APCC}_{\max }(P=0.05)$ and reduction in $\mathrm{APC} \mathrm{T}_{1 / 2}(P=0.02), \mathrm{SN}-38 \mathrm{C}_{\max }(P=0.006)$, and $\mathrm{SN}-38$ glucuronide $\mathrm{T}_{1 / 2}(P=0.04)$. This did not translate into significant alterations in APC, SN38, or SN-38 glucuronide AUC or metabolite/irinotecan AUC ratio $(P>0.05)$.

\section{Pharmacodynamics}

The avid binding of UCN-01 to serum AAG raised the concern of the tissue bioavailability of $\mathrm{UCN}-01$ and potential target inhibition at clinically tolerable doses. Since a key downstream target of PDK1 is p70S6 K, which phosphorylates S6 [37, 63], we hypothesized that if UCN01 inhibited PDK1, we would observe reduced pS6.
Western blot analyses for S6 and pS6 were performed on the peripheral blood mononuclear cells (PBMC) isolated from patient blood (Fig. 1). Representative Western blots of pS6 pre- and post-therapy are shown for three patients (one in Dose Level 4 and 2 in Dose Level 5) and in each case, treatment with UCN-01 (day 3) resulted in a reduction in pS6 but did not alter total levels of S6 (Fig. 1a). Results were analyzed for 23 patient samples (Fig. 1b-d). Importantly, a statistically significant decrease in pS6 was observed on day 3 ( $24 \mathrm{~h}$ following UCN-01 treatment) compared with pre-treatment samples $(P<0.0001)$. Irinotecan treatment alone did not significantly alter pS6 levels $(P=0.29)$. Interestingly, levels of pS6 rose in PBMC by day 8, six days after the first dose of UCN-01, and levels of pS6 between days 1 and 8 were no longer significantly different $(P=0.015)$. Taken together, these results indicate that $\mathrm{UCN}-01$ is bioavailable for at least $24 \mathrm{~h}$ after the first dose but may not be available at therapeutic levels by day 8 . 


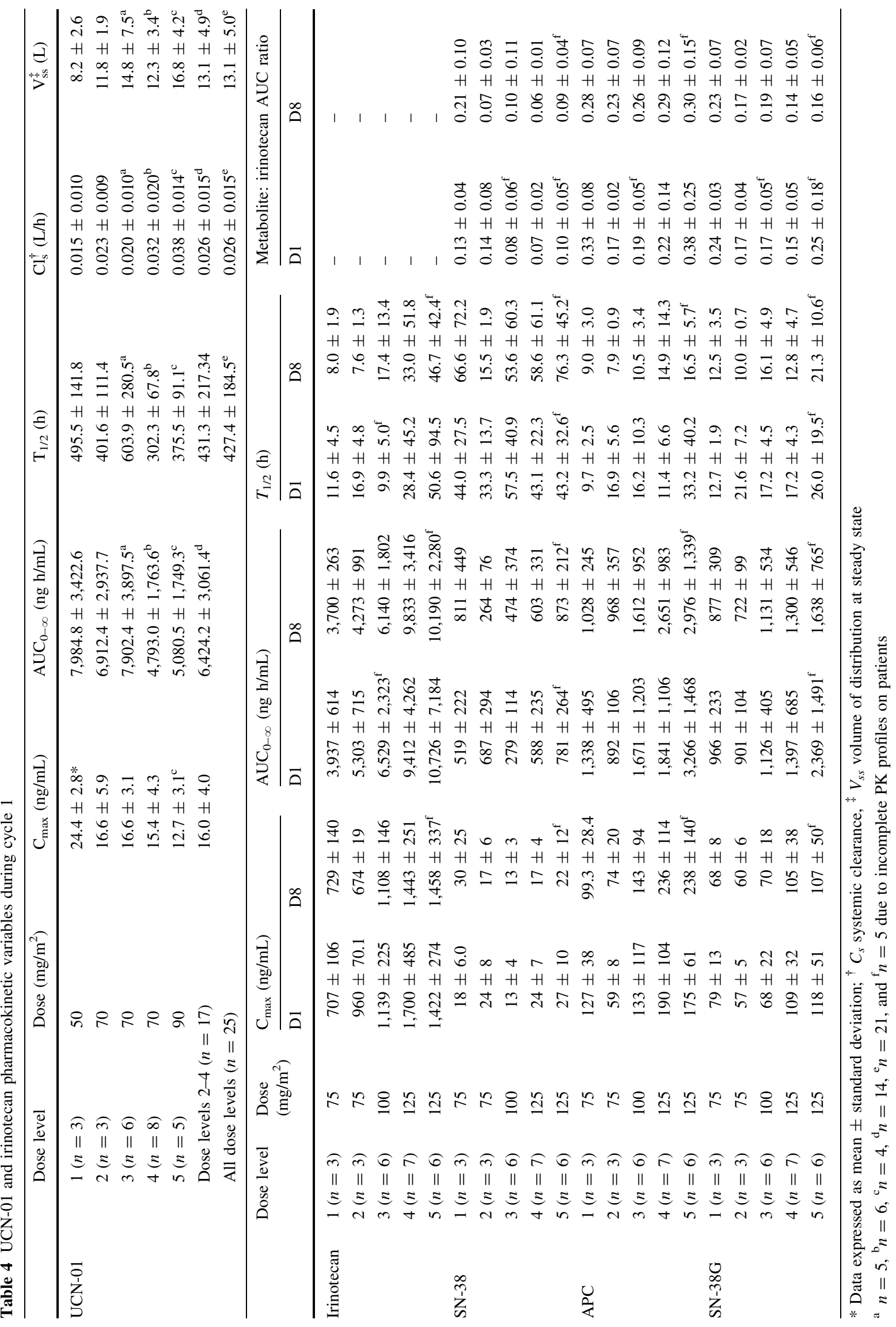




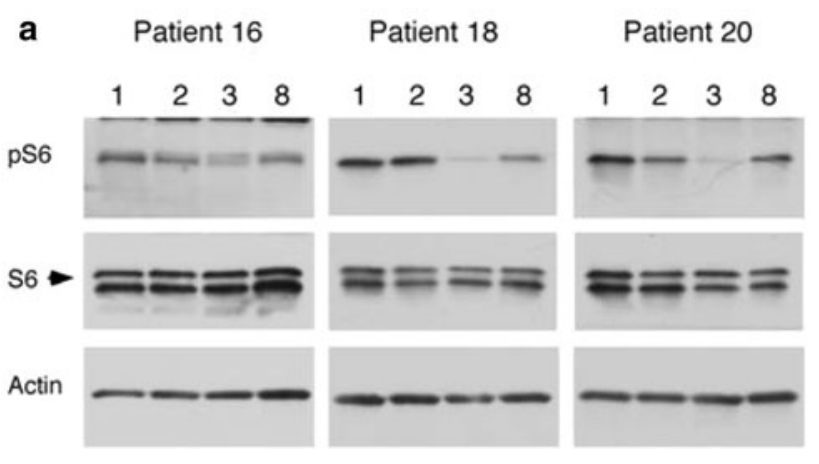

b

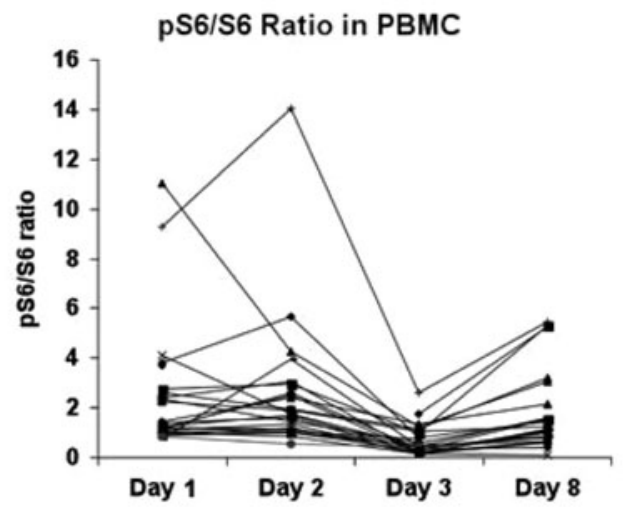

c

\begin{tabular}{|c|c|c|c|}
\hline & DF & t value & P \\
\hline Day 1 vs. 2 & 22 & -1.07 & 0.2952 \\
\hline Day 1 vs. 3 & 23 & 9.51 & $<0.0001^{* *}$ \\
\hline Day 1 vs. 8 & 21 & 2.65 & 0.0150 \\
\hline Day 2 vs. 3 & 22 & 9.53 & $<0.0001^{* *}$ \\
\hline Day 2 vs. 8 & 20 & 3.39 & $0.0029^{* *}$ \\
\hline Day 3 vs. 8 & 21 & -7.40 & $<0.0001^{* *}$ \\
\hline
\end{tabular}

d

\begin{tabular}{|c|c|c|c|}
\hline Day & N & Geometric Mean & $95 \% \mathrm{Cl}$ \\
\hline 1 & 24 & 1.82 & $1.34-2.48$ \\
\hline 2 & 23 & 1.95 & $1.44-2.65$ \\
\hline 3 & 24 & 0.49 & $0.34-0.70$ \\
\hline 8 & 22 & 1.29 & $0.86-1.92$ \\
\hline
\end{tabular}

Fig. 1 Significant decrease in pS6 levels in PBMC following UCN01 treatment. PBMC were collected at baseline (day 1), $24 \mathrm{~h}$ postirinotecan but prior to UCN-01 (day 2), $24 \mathrm{~h}$ post-UCN-01 treatment (day 3 ) and on day 8 prior to the second irinotecan treatment during cycle 1. PBMC were lysed and analyzed by Western blotting with antibodies specific for S6 ribosomal protein, phosphorylated S6 ribosomal protein (pS6), and actin as a loading control. Representative Western blots of PBMC from three patients are shown (a). The arrow indicates total S6 used for normalization. The ratio of phosphorylated S6 to total S6 protein was plotted for each sample at each time point (b). A natural log transformation of the pS6/S6 ratio was required for a normal distribution for the application of standard parametric tests. After log transformation, a paired $t$-test (c) and one-way ANOVA (d) were used to assess the differences among different time points (days 1, 2, 3, 8). Homogeneity of pS6/S6 ratio variance was assessed by Leveve's test, and the variances were found to be equal. A $P$-value of $<0.05$ is considered significant and is indicated by $* *$. $D F$ degrees of freedom, $N$ number of samples, CI confidence interval

Immunohistochemistry (IHC) of phosphorylated S6 was performed on paired rectal biopsy samples (baseline and $24 \mathrm{~h}$ post-UCN-01) from 10 patients in Dose Levels 1-3.
In most cases, the results were not interpretable since sections from paired specimens obtained pre- and posttherapy were often in different orientation in relationship to the intestinal crypts. However, in well-oriented crypts with visible lumens, cytoplasmic pS6 staining was reduced throughout the crypt post-therapy (data not shown). The limited data on pS6 in rectal mucosa support that UCN-01 may be bioavailable in tissue even at the dose levels below that of the MTD.

Serial tumor biopsies were obtained at baseline and $24 \mathrm{~h}$ post-UCN-01 (48 h after irinotecan treatment) from chest wall metastasis in two patients with metastatic breast cancer treated in Dose Level 4. One of these two patients had TNBC and experienced a partial response to therapy for 18 weeks, while the other patient had estrogen and progesterone receptor positive and HER2-negative disease that progressed after one cycle of therapy. As seen in Fig. 2, there was a significant reduction in pS6 staining in both tumor specimens post-treatment (a-d), and $\gamma \mathrm{H} 2 \mathrm{AX}$ staining revealed significantly more DNA double-strand breaks in both tumor samples following the combination treatment $(\mathrm{e}-\mathrm{h})$. These results demonstrate that both irinotecan and UCN-01 were bioavailable in the tumor. Interestingly, two patients with TNBC responded to therapy and both of their tumors were found to have defective p53. Sequencing revealed a missense mutation of CGA $>$ TGA:R306X in one tumor sample (Fig. 2i) and IHC demonstrated nuclear accumulation of p53 in the second tumor sample (Fig. 2j). One patient with ER+ breast cancer progressed after one cycle of therapy, and her tumor was wild type for TP53 (data not shown). These results are consistent with the preclinical findings that UCN-01 works synergistically with DNA-damaging agents in TP53 mutated tumors [3-6, 26, 39, 51, 66, 67]. Immunohistochemical analyses of cleaved caspase 3 and phosphorylated $\mathrm{Cdc} 2$, Chk1, and histone $\mathrm{H} 3$ were performed but the staining was variable and inconclusive.

\section{Efficacy}

All patients, including the 10 patients with resistant, recurrent breast cancer, were evaluated for response (Supplemental Table 1). Two partial responses (8\%) lasting 13 and 18 weeks were observed in women with triplenegative breast cancer (TNBC, estrogen and progesterone receptor negative and HER-2 negative) with chest wall and lymph node recurrences previously treated with an anthracycline, taxane, and capecitabine. Figure $2 \mathrm{k}, 1$ reveal pre- and post-study photos, respectively, of patient 22 , demonstrating resolution of the chest wall lesion after 2 cycles of study treatment. Twelve patients with colorectal (5), breast (4 including 2 with TNBC), pancreatic (1), and prostate (1) cancers and adenocarcinoma of unknown 


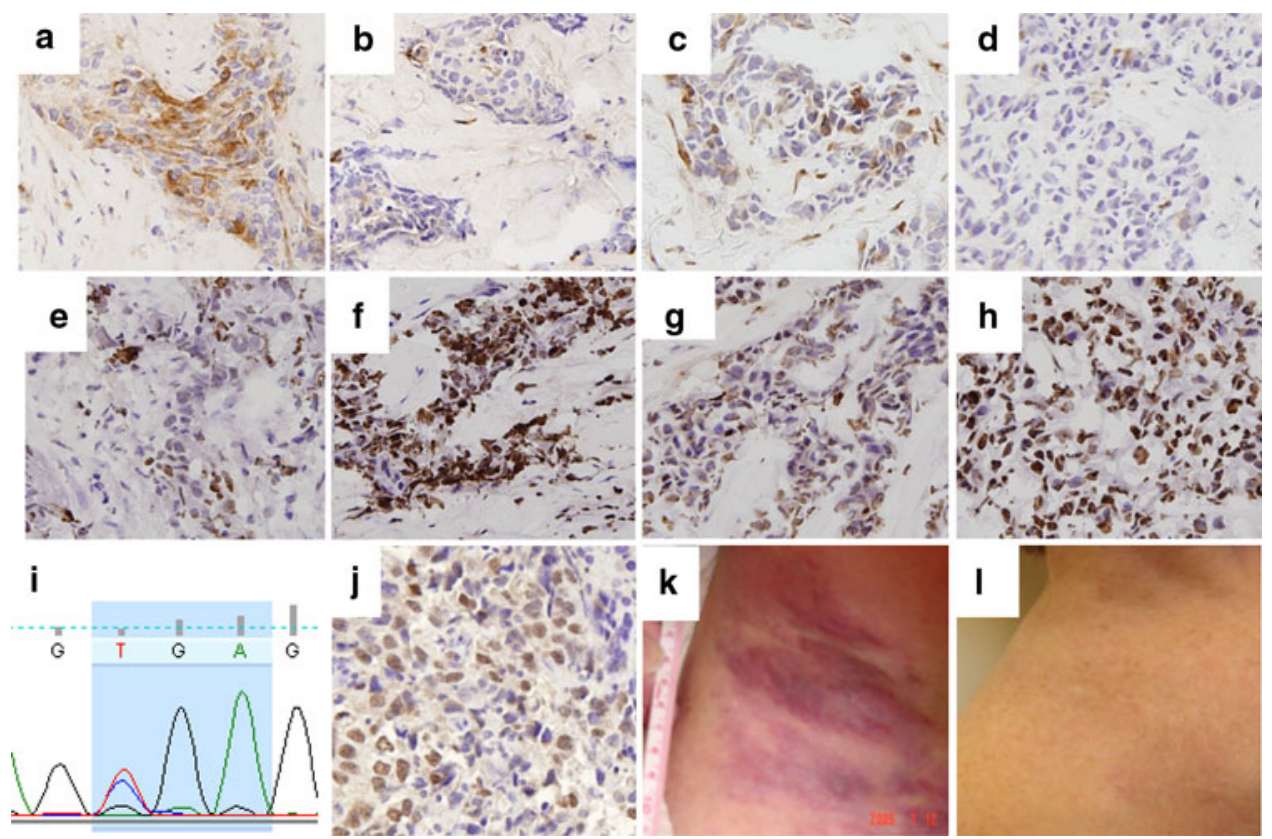

Fig. 2 UCN-01 decreases pS6 in tumor samples and causes a dramatic response of chest wall lesions to therapy. IHC analysis of pS6 on tumor specimens collected at baseline (a) and $24 \mathrm{~h}$ post-UCN01 therapy (b) from Patient 14, whose tumor carried a somatic mutation in TP53 resulting in a change from CGA (Arginine) to TGA (stop codon) at amino acid 306 (i). Patient 14 had TNBC to the chest wall and lymph nodes and a partial response for 18 weeks. IHC analysis of pS6 on tumor specimens collected at baseline (c) and $24 \mathrm{~h}$ post-UCN-01 therapy (d) from Patient 24, whose tumor was ER+, PR+ , HER2 - and was wild type for TP53 by sequencing. Patient 24 had chest wall and lymph nodes metastasis and disease progression after one cycle of study therapy. Representative fields are shown for each specimen. Specimens from Patient 14 had pS6 intensity scores of 2 for both pre-treatment (a) and post-treatment (b), but the percentage of tumor cells positive for pS6 was significantly decreased, from $20 \%$ pre-therapy to $1 \%$ post-therapy. Similarly, specimens from Patient 24 had intensity scores of 3 both pre-treatment (c) and post-therapy (d), but the percentage of tumor cells positive for pS6 was significantly decreased, from $40 \%$ pre-therapy to $5 \%$ post-therapy. IHC analysis of $\gamma \mathrm{H} 2 \mathrm{AX}$ on tumor specimens collected at baseline (e) and $24 \mathrm{~h}$ postUCN-01 therapy (f) from Patient 14 and at baseline (g) and $24 \mathrm{~h}$ postUCN-01 therapy (h) from Patient 24 demonstrated more DNA strand double-strand breaks in both tumor samples following combination treatment. Photographs of metastatic breast cancer to the skin from Patient 22 taken at baseline (k) and following completion of 2 cycles of therapy (l). This tumor exhibited nuclear accumulation of p53 by IHC (j) indicative of mutant TP53. Patient 22 had metastatic TNBC to the skin and lymph nodes and experienced a PR for 13 weeks origin (1) experienced stable disease with the mean duration of 18 weeks (range 7-30 weeks). Eleven patients including 4 with breast cancer (1 with TNBC) had progressive disease (PD).

\section{Discussion}

In this Phase 1 trial of UCN-01 and irinotecan in patients with resistant solid tumor malignancies, we determined the MTD and toxicity profile and examined the pharmacokinetic parameters and target specificity of UCN-01 in vivo. The MTD was defined as Dose Level 4 (irinotecan $125 \mathrm{mg} / \mathrm{m}^{2}$ on days $1,8,15$, and 22 and UCN-01 $70 \mathrm{mg} / \mathrm{m}^{2}$ on day 2 and $35 \mathrm{mg} / \mathrm{m}^{2}$ on day 23 of a 42 -day cycle). The total dose of irinotecan at this MTD was greater than the dose given at the MTD in another Phase 1 study with this combination [22]. In that study, irinotecan $60 \mathrm{mg} / \mathrm{m}^{2}$ on days 1 and 8 and UCN-01 $70 \mathrm{mg} / \mathrm{m}^{2}$ on day 1 and $35 \mathrm{mg} / \mathrm{m}^{2}$ on day 22 of a 21-day cycle was administered. In a 6-week time period at the MTD, patients would have received a total of irinotecan $500 \mathrm{mg} / \mathrm{m}^{2}$ in the 42-day cycle and UCN-01 $240 \mathrm{mg} / \mathrm{m}^{2}$ in two 21 -day cycles. While in our study, two patients were delayed 1 week and subsequently, dose reduced, these patients received a minimum of irinotecan $450 \mathrm{mg} / \mathrm{m}^{2}$. In the study by Jimeno et al. [22], the DLTs were grade 3 hypophosphatemia, grade 4 hyperglycemia, and febrile neutropenia. In our study, the DLTs were grade 3 diarrhea in two patients, one of which had associated grade 3 dehydration, hypokalemia, and hypophosphatemia and grade 3 dyspnea in one patient. Though we observed hypophosphatemia and hyperglycemia, they were not DLTs.

The most common hematologic toxicity was neutropenia with 3 patients experiencing grade 3 neutropenia requiring a treatment delay of a least 1 week accompanied with a dose reduction. The most common non-hematologic toxicities included nausea, vomiting, and diarrhea often associated with dehydration, which were not unexpected. Diarrhea was the dose-limiting toxicity at Dose Level 5. 
Other common toxicities were fatigue, anorexia, and abdominal pain/cramping. Pulmonary toxicity, which has been well documented in previous Phase I trials [47], was also noted in our study. Three patients experienced grade 2-3 dyspnea. The presumed mechanism for the pulmonary toxicity is currently unclear. According to Sausville et al. [47], it is thought that UCN-01 may alter V/Q ratios and possibly allow a functional right-to-left shunt to develop. Eleven patients experienced hyperglycemia, which was self-limiting in all but one patient. This patient, a diabetic on oral hypoglycemic medications, experienced grade 3 hyperglycemia and was hospitalized briefly for treatment and continued on study with closer glucose monitoring. This toxicity has been documented in other studies with this agent [47]. The presumed mechanism for hyperglycemia is thought to be related to inhibition of Akt by UCN01 and resultant changes in glucose transport [29, 47].

There is potential for drug-drug interactions between $\mathrm{UCN}-01$ and irinotecan. The unique pharmacological feature of UCN-01 is a high-affinity binding to human AAG $[15,16,48]$. UCN-01 appears to be eliminated in rats primarily by the liver although the metabolic fate has not been elucidated [32]. Irinotecan has a complex pharmacologic profile with metabolism by human carboxylesterases, cytochrome $\mathrm{P} 450$ enzymes, and UDP-glucuronyltransferases and elimination by several drug-transporting proteins [55]. In addition, irinotecan and the active metabolite $\mathrm{SN}-38$ are bound to albumin, AAG, and $\gamma$-globulins [10]. Similar to results from other Phase I studies, UCN-01 exhibited a long half-life $(427 \mathrm{~h})$, low clearance $(0.026 \mathrm{~L} / \mathrm{h})$, and marked variability in AUC values (3-fold) [12, 22]. As previously observed, there was a correlation between UCN-01 clearance and AAG concentrations [58]. We also observed that UCN-01 exposure appeared to decline with increasing doses of irinotecan, although only $\mathrm{C}_{\max }$ reached statistical significance. This trend may be due to the saturation of protein binding [58] or a drug interaction at the protein binding level. Not unexpectedly, there were no correlations between increasing UCN-01 exposure and increasing grade of toxicity, which is consistent with previous reports $[12,22]$. As was previously observed, there were alterations in the irinotecan pharmacokinetics albeit not the same pharmacokinetic parameters [22]. The mechanism behind this drug interaction is unknown.

Our correlative studies demonstrated decreases in pS6 in PBMC, rectal mucosa, and tumor biopsies after UCN-01 treatment. The number of rectal mucosa and tumor biopsy samples was very limited, and no conclusions could be made. However, the decrease in pS6 was significant in PBMC, indicating that UCN-01 is bioavailable and inhibits PDK1 at the MTD for at least $24 \mathrm{~h}$ after the first dose of $\mathrm{UCN}-01$. Interestingly, this decrease in pS6 was no longer observed in PBMC at day 8 of the treatment. Under the treatment plan, irinotecan was administered on days 1,8 , 15 and 22 and UCN-01 was administered on days 2 and 23 of each 42-day cycle. The correlative studies indicate that UCN-01 may no longer be bioavailable in tumors during subsequent treatments with irinotecan (days 8, 15, and 22). Therefore, this treatment regimen may not be optimal for inducing checkpoint bypass in response to the DNA damage induced by irinotecan.

Previous Phase I studies, either with single agent UCN-01 or in combination with other agents, have shown minimal responses in patients with solid tumor malignancies. Partial responses were seen in a patient with melanoma $[12,48]$, a woman with adenocarcinoma of unknown primary with skin metastases (presumed to be TNBC primary, personal communication with P.N. Lara) [34], and one woman with ovarian cancer [19]. Interestingly, our study demonstrates two partial responses in women with TNBC and both of their tumors were defective in p53. Of the 12 patients with stable disease, four had breast cancer (2 with TNBC). Given that the prognosis for women with TNBC is poor due to the aggressive characteristics of their tumors and limited treatment options [20,53], the development of more effective therapies is a high priority. TNBC typically falls into the basal-like subtype when examined by DNA microarray analysis [38]. Patients with basal-like breast cancer have a significantly shorter survival in comparison with patients with luminal (ER+) subtypes [38, 56, 57]. Interestingly, a much higher rate of TP53 mutations (44\% in basal-like vs. $15 \%$ in luminal A subtype, $P<0.001$ ) [8] and loss of PTEN (67\% in ER-/PgR- vs. $23 \%$ in ER+ $/ \mathrm{PgR}+, P<0.05)$ have been observed [11, 33, 42, 44]. Theoretically, agents such as UCN-01 that target proteins in both pathways may prove to be particularly effective in a dual TP53 and PTEN mutant tumor. Therefore, based on the preliminary results obtained in our small subset of women with TNBC, the NCI CTEP approved an extension of our study (http://clinicaltrials.gov, NCT00031681) to determine the efficacy and tolerability of this combination in these women after failure of anthracyclines and taxanes therapy. In addition, pharmacodynamic parameters including TP53 mutational status and cell cycle, checkpoint, and signaling proteins will be correlated with response.

The cellular targets of $\mathrm{UCN}-01$ include protein kinase $\mathrm{C}$ isoforms, CDKs, Chk1, and PDK1. These targets likely account for the varied toxicities observed in clinical trials. Because of these toxicities, it is unclear whether UCN-01 will undergo further development. Nonetheless, more selective checkpoint kinase inhibitors are in clinical trials now. Over 33 patent applications have been filed from January 2006 through August 2008, claiming chemical matter in which Chk1 or Chk2 were stated as targets of inhibition [21]. Given the plethora of compounds that may 
be placed into clinical trials, it is imperative that "proof of concept" trials be performed on this important class of compounds.

Acknowledgments We wish to thank the patients and their families for participation in this study. We also thank the nurses, clinical research and regulatory coordinators at the Siteman Cancer Center for their care of the patients on this study. Dr. Mark A. Watson, Director, Tissue Procurement Core, Alvin J. Siteman Cancer Center at Washington University School of Medicine and Barnes-Jewish Hospital is thanked for tissue acquisition, storage, and processing, and Dr. Katherine Deschryver is thanked for IHC scoring. Grant support: St. Louis Men's Group Against Cancer and NCI Translational Research Initiative Subcontract 22XS046 (P. M. Fracasso), P30 CA091842 (Pharmacology Core, Alvin J. Siteman Cancer Center), Doris Duke Charitable Foundation (R.C. Chen), P30 CA069773 (Analytical Pharmacology Core, Sidney Kimmel Comprehensive Cancer Center at Johns Hopkins), Howard Hughes Medical Institute, Komen Foundation, UL1 RR024992.

Conflict of interest No potential conflict of interest was disclosed.

Open Access This article is distributed under the terms of the Creative Commons Attribution Noncommercial License which permits any noncommercial use, distribution, and reproduction in any medium, provided the original author(s) and source are credited.

\section{References}

1. Abe K, Yoshida M, Usui T, Horinouchi S, Beppu T (1991) Highly synchronous culture of fibroblasts from G2 block caused by staurosporine, a potent inhibitor of protein kinases. Exp Cell Res 192:122-127

2. Abramoff MD (2001) Roaming through methodology XXIX. P. Ned Tijdschr Geneeskd 145:602

3. Akinaga S, Nomura K, Gomi K, Okabe M (1994) Effect of $\mathrm{UCN}-01$, a selective inhibitor of protein kinase $\mathrm{C}$, on the cellcycle distribution of human epidermoid carcinoma, A431 cells. Cancer Chemother Pharmacol 33:273-280

4. Akiyama T, Shimizu M, Okabe M, Tamaoki T, Akinaga S (1999) Differential effects of UCN-01, staurosporine and CGP 41251 on cell cycle progression and CDC2/cyclin B1 regulation in A431 cells synchronized at $\mathrm{M}$ phase by nocodazole. Anticancer Drugs 10:67-78

5. Bredel M, Pollack IF, Freund JM, Rusnak J, Lazo JS (1999) Protein kinase $\mathrm{C}$ inhibition by UCN-01 induces apoptosis in human glioma cells in a time-dependent fashion. J Neurooncol 41:9-20

6. Bunch RT, Eastman A (1996) Enhancement of cisplatin-induced cytotoxicity by 7-hydroxystaurosporine (UCN-01), a new G2checkpoint inhibitor. Clin Cancer Res 2:791-797

7. Busby EC, Leistritz DF, Abraham RT, Karnitz LM, Sarkaria JN (2000) The radiosensitizing agent 7-hydroxystaurosporine (UCN01) inhibits the DNA damage checkpoint kinase hChk1. Cancer Res 60:2108-2112

8. Carey LA, Perou CM, Livasy CA, Dressler LG, Cowan D, Conway K, Karaca G, Troester MA, Tse CK, Edmiston S, Deming SL, Geradts J, Cheang MC, Nielsen TO, Moorman PG, Earp HS, Millikan RC (2006) Race, breast cancer subtypes, and survival in the Carolina breast cancer study. Jama 295:2492-2502

9. Cheson BD, Zwiebel JA, Dancey J, Murgo A (2000) Novel therapeutic agents for the treatment of myelodysplastic syndromes. Semin Oncol 27:560-577
10. Combes O, Barre J, Duche JC, Vernillet L, Archimbaud Y, Marietta MP, Tillement JP, Urien S (2000) In vitro binding and partitioning of irinotecan (CPT-11) and its metabolite, $\mathrm{SN}-38$, in human blood. Invest New Drugs 18:1-5

11. Crowder RJ, Ellis MJ (2005) Treating breast cancer through novel inhibitors of the phosphatidylinositol $3^{\prime}$-kinase pathway. Breast Cancer Res 7:212-214

12. Dees EC, Baker SD, O'Reilly S, Rudek MA, Davidson SB, Aylesworth C, Elza-Brown K, Carducci MA, Donehower RC (2005) A phase I and pharmacokinetic study of short infusions of UCN-01 in patients with refractory solid tumors. Clin Cancer Res 11:664-671

13. Eastman A (2004) Cell cycle checkpoints and their impact on anticancer therapeutic strategies. J Cell Biochem 91:223231

14. Edelman MJ, Bauer KS Jr, Wu S, Smith R, Bisacia S, Dancey J (2007) Phase I and pharmacokinetic study of 7-Hydroxystaurosporine and Carboplatin in advanced solid tumors. Clin Cancer Res 13:2667-2674

15. Fuse E, Tanii H, Kurata N, Kobayashi H, Shimada Y, Tamura T, Sasaki Y, Tanigawara Y, Lush RD, Headlee D, Figg WD, Arbuck SG, Senderowicz AM, Sausville EA, Akinaga S, Kuwabara T, Kobayashi S (1998) Unpredicted clinical pharmacology of UCN01 caused by specific binding to human alpha1-acid glycoprotein. Cancer Res 58:3248-3253

16. Fuse E, Tanii H, Takai K, Asanome K, Kurata N, Kobayashi H, Kuwabara T, Kobayashi S, Sugiyama Y (1999) Altered pharmacokinetics of a novel anticancer drug, UCN-01, caused by specific high affinity binding to alpha1-acid glycoprotein in humans. Cancer Res 59:1054-1060

17. Gibaldi M, Perrier D (1982) Noncompartmental analysis based on statistical moment theory. Pharmacokinetics New York, NY, pp 409-417

18. Graves PR, Yu L, Schwarz JK, Gales J, Sausville EA, O’Connor PM, Piwnica-Worms H (2000) The Chk1 protein kinase and the $\mathrm{Cdc} 25 \mathrm{C}$ regulatory pathways are targets of the anticancer agent UCN-01. J Biol Chem 275:5600-5605

19. Hotte SJ, Oza A, Winquist EW, Moore M, Chen EX, Brown S, Pond GR, Dancey JE, Hirte HW (2006) Phase I trial of UCN-01 in combination with topotecan in patients with advanced solid cancers: a Princess Margaret Hospital Phase II Consortium study. Ann Oncol 17:334-340

20. Howell A, Cuzick J, Baum M, Buzdar A, Dowsett M, Forbes JF, Hoctin-Boes G, Houghton J, Locker GY, Tobias JS (2005) Results of the ATAC (Arimidex, Tamoxifen, Alone or in Combination) trial after completion of 5 years' adjuvant treatment for breast cancer. Lancet 365:60-62

21. Janetka JW, Ashwell S (2009) Checkpoint kinase inhibitors: a review of the patent literature. Expert Opinion on Therapeutic Patents 19:165-197

22. Jimeno A, Rudek MA, Purcell T, Laheru DA, Messersmith WA, Dancey J, Carducci MA, Baker SD, Hidalgo M, Donehower RC (2008) Phase I and pharmacokinetic study of UCN-01 in combination with irinotecan in patients with solid tumors. Cancer Chemother Pharmacol 61:423-433

23. Jones CB, Clements MK, Redkar A, Daoud SS (2000) UCN-01 and camptothecin induce DNA double-strand breaks in p53 mutant tumor cells, but not in normal or p53 negative epithelial cells. Int J Oncol 17:1043-1051

24. Jones CB, Clements MK, Wasi S, Daoud SS (2000) Enhancement of camptothecin-induced cytotoxicity with UCN-01 in breast cancer cells: abrogation of $\mathrm{S} / \mathrm{G}(2)$ arrest. Cancer Chemother Pharmacol 45:252-258

25. Kastan MB, Onyekwere O, Sidransky D, Vogelstein B, Craig RW (1991) Participation of p53 protein in the cellular response to DNA damage. Cancer Res 51:6304-6311 
26. Kawakami K, Futami H, Takahara J, Yamaguchi K (1996) UCN01, 7-hydroxyl-staurosporine, inhibits kinase activity of cyclindependent kinases and reduces the phosphorylation of the retinoblastoma susceptibility gene product in A549 human lung cancer cell line. Biochem Biophys Res Commun 219:778-783

27. Koh J, Kubota T, Migita T, Abe S, Hashimoto M, Hosoda Y, Kitajima M (2002) UCN-01 (7-hydroxystaurosporine) inhibits the growth of human breast cancer xenografts through disruption of signal transduction. Breast Cancer 9:50-54

28. Kohn EA, Yoo CJ, Eastman A (2003) The protein kinase C inhibitor Go6976 is a potent inhibitor of DNA damage-induced $S$ and G2 cell cycle checkpoints. Cancer Res 63:31-35

29. Kondapaka SB, Zarnowski M, Yver DR, Sausville EA, Cushman SW (2004) 7-Hydroxystaurosporine (UCN-01) Inhibition of Akt Thr308 but not Ser473 phosphorylation. Clin Cancer Res 10:7192-7198

30. Kortmansky J, Shah MA, Kaubisch A, Weyerbacher A, Yi S, Tong W, Sowers R, Gonen M, O'Reilly E, Kemeny N, Ilson DI, Saltz LB, Maki RG, Kelsen DP, Schwartz GK (2005) Phase I trial of the cyclin-dependent kinase inhibitor and protein kinase $\mathrm{C}$ inhibitor 7-hydroxystaurosporine in combination with Fluorouracil in patients with advanced solid tumors. J Clin Oncol 23:1875-1884

31. Kucab JE, Lee C, Chen CS, Zhu J, Gilks CB, Cheang M, Huntsman D, Yorida E, Emerman J, Pollak M, Dunn SE (2005) Celecoxib analogues disrupt Akt signaling, which is commonly activated in primary breast tumours. Breast Cancer Res 7:R796R807

32. Kurata N, Kuwabara T, Tanii H, Fuse E, Akiyama T, Akinaga S, Kobayashi H, Yamaguchi K, Kobayashi S (1999) Pharmacokinetics and pharmacodynamics of a novel protein kinase inhibitor, UCN-01. Cancer Chemother Pharmacol 44:12-18

33. Kurose K, Gilley K, Matsumoto S, Watson PH, Zhou XP, Eng C (2002) Frequent somatic mutations in PTEN and TP53 are mutually exclusive in the stroma of breast carcinomas. Nat Genet 32:355-357

34. Lara PN Jr, Mack PC, Synold T, Frankel P, Longmate J, Gumerlock PH, Doroshow JH, Gandara DR (2005) The cyclindependent kinase inhibitor UCN-01 plus cisplatin in advanced solid tumors: a California cancer consortium phase I pharmacokinetic and molecular correlative trial. Clin Cancer Res 11:4444-4450

35. Levesque AA, Eastman A (2007) p53-based cancer therapies: Is defective p53 the Achilles heel of the tumor? Carcinogenesis 28:13-20

36. Levesque AA, Kohn EA, Bresnick E, Eastman A (2005) Distinct roles for $\mathrm{p} 53$ transactivation and repression in preventing $\mathrm{UCN}$ 01-mediated abrogation of DNA damage-induced arrest at S and G2 cell cycle checkpoints. Oncogene 24:3786-3796

37. Mora A, Komander D, van Aalten DM, Alessi DR (2004) PDK1, the master regulator of AGC kinase signal transduction. Semin Cell Dev Biol 15:161-170

38. Nielsen TO, Hsu FD, Jensen K, Cheang M, Karaca G, Hu Z, Hernandez-Boussard T, Livasy C, Cowan D, Dressler L, Akslen LA, Ragaz J, Gown AM, Gilks CB, van de Rijn M, Perou CM (2004) Immunohistochemical and clinical characterization of the basal-like subtype of invasive breast carcinoma. Clin Cancer Res 10:5367-5374

39. Nieves-Neira W, Pommier Y (1999) Apoptotic response to camptothecin and 7-hydroxystaurosporine $(\mathrm{UCN}-01)$ in the 8 human breast cancer cell lines of the NCI Anticancer Drug Screen: multifactorial relationships with topoisomerase I, protein kinase C, Bcl-2, p53, MDM-2 and caspase pathways. Int J Cancer 82:396-404

40. Patel V, Lahusen $\mathrm{T}$, Leethanakul $\mathrm{C}$, Igishi $\mathrm{T}$, Kremer $\mathrm{M}$, Quintanilla-Martinez L, Ensley JF, Sausville EA, Gutkind JS,
Senderowicz AM (2002) Antitumor activity of UCN-01 in carcinomas of the head and neck is associated with altered expression of cyclin D3 and p27(KIP1). Clin Cancer Res 8:3549-3560

41. Perez RP, Lewis LD, Beelen AP, Olszanski AJ, Johnston N, Rhodes CH, Beaulieu B, Ernstoff MS, Eastman A (2006) Modulation of cell cycle progression in human tumors: a pharmacokinetic and tumor molecular pharmacodynamic study of cisplatin plus the Chk1 inhibitor UCN-01 (NSC 638850). Clin Cancer Res 12:7079-7085

42. Perren A, Weng LP, Boag AH, Ziebold U, Thakore K, Dahia PL, Komminoth P, Lees JA, Mulligan LM, Mutter GL, Eng C (1999) Immunohistochemical evidence of loss of PTEN expression in primary ductal adenocarcinomas of the breast. Am J Pathol 155:1253-1260

43. Rivory LP, Robert J (1994) Reversed-phase high-performance liquid chromatographic method for the simultaneous quantitation of the carboxylate and lactone forms of the camptothecin derivative irinotecan, CPT-11, and its metabolite SN-38 in plasma. J Chromatogr B Biomed Appl 661:133-141

44. Saal LH, Holm K, Maurer M, Memeo L, Su T, Wang X, Yu JS, Malmstrom PO, Mansukhani M, Enoksson J, Hibshoosh H, Borg A, Parsons R (2005) PIK3CA mutations correlate with hormone receptors, node metastasis, and ERBB2, and are mutually exclusive with PTEN loss in human breast carcinoma. Cancer Res 65:2554-2559

45. Sampath D, Cortes J, Estrov Z, Du M, Shi Z, Andreeff M, Gandhi V, Plunkett W (2006) Pharmacodynamics of cytarabine alone and in combination with 7-hydroxystaurosporine (UCN01) in AML blasts in vitro and during a clinical trial. Blood 107:2517-2524

46. Sato S, Fujita N, Tsuruo T (2002) Interference with PDK1-Akt survival signaling pathway by UCN-01 (7-hydroxystaurosporine). Oncogene 21:1727-1738

47. Sausville EA, Arbuck SG, Messmann R, Headlee D, Bauer KS, Lush RM, Murgo A, Figg WD, Lahusen T, Jaken S, Jing X, Roberge M, Fuse E, Kuwabara T, Senderowicz AM (2001) Phase I trial of $72 \mathrm{~h}$ continuous infusion UCN-01 in patients with refractory neoplasms. J Clin Oncol 19:2319-2333

48. Sausville EA, Lush RD, Headlee D, Smith AC, Figg WD, Arbuck SG, Senderowicz AM, Fuse E, Tanii H, Kuwabara T, Kobayashi S (1998) Clinical pharmacology of UCN-01: initial observations and comparison to preclinical models. Cancer Chemother Pharmacol 42(Suppl):S54-S59

49. Senderowicz AM (2001) Development of cyclin-dependent kinase modulators as novel therapeutic approaches for hematological malignancies. Leukemia 15:1-9

50. Senderowicz AM, Sausville EA (2000) Preclinical and clinical development of cyclin-dependent kinase modulators. J Natl Cancer Inst 92:376-387

51. Seynaeve CM, Stetler-Stevenson M, Sebers S, Kaur G, Sausville EA, Worland PJ (1993) Cell cycle arrest and growth inhibition by the protein kinase antagonist UCN-01 in human breast carcinoma cells. Cancer Res 53:2081-2086

52. Shao RG, Cao CX, Shimizu T, O'Connor PM, Kohn KW, Pommier Y (1997) Abrogation of an S-phase checkpoint and potentiation of camptothecin cytotoxicity by 7-hydroxystaurosporine (UCN-01) in human cancer cell lines, possibly influenced by p53 function. Cancer Res 57:4029-4035

53. Slamon DJ, Leyland-Jones B, Shak S, Fuchs H, Paton V, Bajamonde A, Fleming T, Eiermann W, Wolter J, Pegram M, Baselga J, Norton L (2001) Use of chemotherapy plus a monoclonal antibody against HER2 for metastatic breast cancer that overexpresses HER2. N Engl J Med 344:783-792

54. Slichenmyer WJ, Rowinsky EK, Donehower RC, Kaufmann SH (1993) The current status of camptothecin analogues as antitumor agents. J Natl Cancer Inst 85:271-291 
55. Smith NF, Figg WD, Sparreboom A (2006) Pharmacogenetics of irinotecan metabolism and transport: an update. Toxicol In Vitro 20:163-175

56. Sorlie T, Perou CM, Tibshirani R, Aas T, Geisler S, Johnsen H, Hastie T, Eisen MB, van de Rijn M, Jeffrey SS, Thorsen T, Quist H, Matese JC, Brown PO, Botstein D, Eystein Lonning P, Borresen-Dale AL (2001) Gene expression patterns of breast carcinomas distinguish tumor subclasses with clinical implications. Proc Natl Acad Sci U S A 98:10869-10874

57. Sorlie T, Tibshirani R, Parker J, Hastie T, Marron JS, Nobel A, Deng S, Johnsen H, Pesich R, Geisler S, Demeter J, Perou CM, Lonning PE, Brown PO, Borresen-Dale AL, Botstein D (2003) Repeated observation of breast tumor subtypes in independent gene expression data sets. Proc Natl Acad Sci USA 100: 8418-8423

58. Sparreboom A, Chen H, Acharya MR, Senderowicz AM, Messmann RA, Kuwabara T, Venzon DJ, Murgo AJ, Headlee D, Sausville EA, Figg WD (2004) Effects of alpha1-acid glycoprotein on the clinical pharmacokinetics of 7-hydroxystaurosporine. Clin Cancer Res 10:6840-6846

59. Takahashi I, Kobayashi E, Asano K, Yoshida M, Nakano H (1987) UCN-01, a selective inhibitor of protein kinase C from streptomyces. J Antibiot (Tokyo) 40:1782-1784

60. Takahashi I, Kobayashi E, Nakano H, Murakata C, Saitoh H, Suzuki K, Tamaoki T (1990) Potent selective inhibition of 7-Omethyl UCN-01 against protein kinase C. J Pharmacol Exp Ther 255:1218-1221

61. Takahashi I, Saitoh Y, Yoshida M, Sano H, Nakano H, Morimoto M, Tamaoki T (1989) UCN-01 and UCN-02, new selective inhibitors of protein kinase C. II. Purification, physico-chemical properties, structural determination and biological activities. J Antibiot (Tokyo) 42:571-576
62. Tamaoki T, Nakano H (1990) Potent and specific inhibitors of protein kinase $\mathrm{C}$ of microbial origin. Biotechnology (NY) $8: 732-735$

63. Templeton DJ (2001) Protein kinases: getting NEKed for S6 K activation. Curr Biol 11:R596-R599

64. Therasse P, Arbuck SG, Eisenhauer EA, Wanders J, Kaplan RS, Rubinstein L, Verweij J, Van Glabbeke M, van Oosterom AT, Christian MC, Gwyther SG (2000) New guidelines to evaluate the response to treatment in solid tumors. European organization for research and treatment of cancer, national cancer institute of the United States, national cancer institute of Canada. J Natl Cancer Inst 92:205-216

65. Tse AN, Schwartz GK (2004) Potentiation of Cytotoxicity of Topoisomerase I poison by concurrent and sequential treatment with the checkpoint inhibitor UCN-01 involves disparate mechanisms resulting in either p53-independent clonogenic suppression or p53-dependent mitotic catastrophe. Cancer Res 64: $6635-6644$

66. Usuda J, Saijo N, Fukuoka K, Fukumoto H, Kuh HJ, Nakamura T, Koh Y, Suzuki T, Koizumi F, Tamura T, Kato H, Nishio K (2000) Molecular determinants of UCN-01-induced growth inhibition in human lung cancer cells. Int J Cancer 85:275-280

67. Wang Q, Fan S, Eastman A, Worland PJ, Sausville EA, O'Connor PM (1996) UCN-01: a potent abrogator of G2 checkpoint function in cancer cells with disrupted p53. J Natl Cancer Inst 88:956-965

68. Yu L, Orlandi L, Wang P, Orr MS, Senderowicz AM, Sausville EA, Silvestrini R, Watanabe N, Piwnica-Worms H, O'Connor PM (1998) UCN-01 abrogates G2 arrest through a Cdc2-dependent pathway that is associated with inactivation of the Wee1Hu kinase and activation of the Cdc25C phosphatase. J Biol Chem 273:33455-33464 